\title{
К ТЕОРИИ НЕОДНОРОДНЫХ ОПТИЧЕСКИХ ПЛЕНОК ПРИ НАКЛОННОМ ПАДЕНИИ СВЕТА
}

P. KARD. MITTEHOMOGEENSETE OPTILISTE KILEDE TEOORIAST VALGUSE KALDU LANGEMISE PUHUL

P. KARD. ON THE THEORY OF THE INHOMOGENEOUS OPTICAL FILMS IN THE CASE OF OBLIQUE INCIDENCE OF LIGHT

В предыдущей статье $\left[{ }^{1}\right]$ был предложен новый метод решения одномерных волновых уравнений, расширяющий круг уравнений, имеющих решение в замкнутом виде. Там же этот метод был применен к нахождению матрицы интерференции неоднородной пленки при нормальном падении света. В настоящем сообщении мы рассмотрим возможности этого метода в случае наклонного падения света, поляризованного перпендикулярно плоскости падения.

Волновое уравнение имеет в этом случае вид

$$
d^{2} U / d z^{2}+k^{2}\left(n^{2}(z)-p^{2}\right) U=0,
$$

где

$$
p=n_{0} \sin \vartheta_{0},
$$

$n_{0}$ - показатель преломления исходной среды и $\vartheta_{0}$ - угол падения. Положим

и

$$
\begin{gathered}
s=n^{1 / 2}, \\
u=\int n(z) d z
\end{gathered}
$$

$$
A=s U \text {. }
$$

Тогда уравнение (1) принимает вид:

$$
A^{-1} d^{2} A / d u^{2}-s^{-1} d^{2} s / d u^{2}+k^{2}\left(1-p^{2} s^{-4}\right)=0 .
$$

Если положим

$$
d^{2} s / d u^{2}+f(u) s=0,
$$

то $A$ удовлетворяет уравнению

$$
d^{2} A / d u^{2}+\left[f(u)+k^{2}\left(1-p^{2} s^{-4}\right)\right] A=0 .
$$

Таким образом, уравнение (1) сведено к системе уравнений (7) и (8). Если при некоторой заданной функции $f(u)$ оба эти уравнения имеют 
решения в замкнутом виде, то, обозначив одно из решений уравнения (7) через $s_{1}$, получим замкнутое решение уравнения (1), согласно формуле (5), в виде

$$
U=s_{1}^{-1} A .
$$

Зависимость показателя преломления $n$ от координаты $z$ определяется формулами

$$
n=s_{1}^{2}
$$

H

$$
z=\sigma^{-1}\left(s_{2} / s_{1}\right)+\text { const, }
$$

вытекающими из формул (3) и (4). В формуле (11) $s_{2}$ - линейно независимое от $s_{1}$ решение уравнения $(7)$, а

$$
\sigma=s_{1} d s_{2} / d u-s_{2} d s_{1} / d u
$$

- его вронскиан.

В отличие от случая нормального падения света изложенный метод не привел до сих пор для наклонного падения к обнаружению новых примеров уравнений вида (1), разрешимых в замкнутой форме. Тем не менее метод заслуживает внимания как путь возможного обнаружения подобных примеров в будушем. Помимо этого, он может быть полезен для исследования уже известных уравнений. Приведем как иллюстрацию сказанного один пример. Примем в уравнении (1)

$$
n(z)=a^{2}(1+b z / h)^{-1},
$$

где $h$ - толщина слоя, а $a$ и $b$ - безразмерные числа. Для решения волнового уравнения

$$
d^{2} U / d z^{2}+k^{2}\left[\frac{a^{4}}{(1+b z / h)^{2}}-p^{2}\right] U=0
$$

возьмем

$$
f=-b^{2} / 4 a^{4} h^{2} \text {. }
$$

Тогда уравнение (7) получит вид

$$
d^{2} s / d u^{2}-\left(b^{2} / 4 a^{4} h^{2}\right) s=0,
$$

решения которого примем в виде:

$$
\begin{aligned}
& s_{1}=a \exp \left(-b u / 2 a^{2} h\right), \\
& s_{2}=a \exp \left(b u / 2 a^{2} h\right) .
\end{aligned}
$$

Отсюда

$$
\sigma=b / h
$$

и, согласно формуле (11),

$$
z=(h / b)\left[\exp \left(b u / a^{2} h\right)-1\right],
$$

а в силу формулы (10)

$$
n=a^{2} \exp \left(-b u / a^{2} h\right) .
$$

Исключая $u$ из формул (19) и (20), находим

$$
n(z)=a^{2}(1+b z / h)^{-1},
$$

что совпадает с заданной формулой (13). Остается решить уравнение (8), принимающее в данном случае вид 


$$
d^{2} A / d u^{2}+\left[k^{2}-b^{2} / 4 a^{4} h^{2}-\left(k^{2} p^{2} / a^{4}\right) \exp \left(2 b u / a^{2} h\right)\right] A=0 .
$$

Это уравнение сводится, как известно, к уравнению Бесселя и его решение таково:

$$
A(u)=Z_{v}\left((i k h p / b) \exp \left(b u / a^{2} h\right)\right),
$$

где $Z_{v}-$ функция Бесселя порядка $v$ и

$$
v=\left(\frac{1}{4}-\frac{a^{4} h^{2} k^{2}}{b^{2}}\right)^{1 / 2}
$$

Окончательно получаем решение уравнения (14), согласно формулам (9), (17), (19) и (22), в виде

$$
U(z)=(1+b z / h)^{1 / 2} Z_{v}((i k h p / b)(1+b z / h)) .
$$

\section{Л ИТ Е Р А Т У Р А}

1. К ард П. Об одномерных волновых уравнениях, решаемых в замкнутом виде. - Изв. АН ЭССР, Физ. Матем., 1977, т. 26, № 3, с. 252-259.

\section{Тартуский государственный} университет
Поступила в редакцию 6/II 1978 